\title{
Le Modèle de Leadership Pour Une Conduite Efficiente de Changement dans les Organisations Internationales en Afrique Subsaharienne Francophone
}

\author{
Dounia Bere M., \\ Doctorant en Sociologie des Organisations et de la Gouvernance, \\ Université de l'Alliance Chrétienne d'Abidjan (UACA), Côte d'Ivoire \\ Kouadio Patrick Adon, \\ Maître de Conférences en Sociologie, \\ Université Félix Houphouët-Boigny d'Abidjan
}

Doi:10.19044/esj.2019.v15n25p143 URL:http://dx.doi.org/10.19044/esj.2019.v15n25p143

\section{Résumé}

L'objectif général de cette recherche multidisciplinaire est l'évaluation de l'impact des traits culturels de l'Afrique subsaharienne francophone sur le « leadership » en général, et en particulier, lorsqu'il est exercé dans un contexte de changement organisationnel. La recherche a été faite sur des processus de réduction d'effectifs à OCHA Côte d'Ivoire (2013-2014) et à ICRISAT Niger (2015), à partir d'une perspective épistémologique multiparadigmatique (constructivisme et empirisme). Une analyse qualitative des données primaires et secondaires recueillies sur les deux processus a abouti à des résultats qui ont confirmé la théorie culturelle du leadership à dominante humaine attribuée par l'étude GLOBE à la zone considérée. Les caractéristiques identifiées des styles de leadership qui ont été instrumentaux au succès de ces processus ont inspiré la principale contribution théorique de cette recherche : le modèle de leadership SMART, empreint d'EMPATHIE.

Mots clés: Culture, Leadership, Changement Organisationnel, Réduction d'effectifs, Organisation, Afrique subsaharienne francophone 


\title{
The Leadership Model for Efficient Driving Change in International Organizations in Sub-Saharan Africa
}

\author{
Dounia Bere M., \\ Doctorant en Sociologie des Organisations et de la Gouvernance, \\ Université de l'Alliance Chrétienne d'Abidjan (UACA), Côte d'Ivoire \\ Kouadio Patrick Adon, \\ Maître de Conférences en Sociologie, \\ Université Félix Houphouët-Boigny d'Abidjan
}

\begin{abstract}
The overall objective of this multidisciplinary research is to assess the impact of the cultural traits of the French-speaking sub-Saharan Africa on "leadership" in general, and in particular when exercised in a context of organizational change. The research was done on downsizing processes in OCHA Côte d'Ivoire (2013-2014) and ICRISAT Niger (2015), from a multiparadigmatic epistemological perspective (constructivism and empiricism). A qualitative analysis of the primary and secondary data collected on the two processes has yielded results that have confirmed the human dominated cultural theory of leadership attributed by the GLOBE study to the considered area. The characteristics of the leadership styles identified as instrumental in the success of these processes has inspired the main theoretical contribution of the research which is the EMPATHIC and SMART Leadership Model.
\end{abstract}

Keywords: Culture, Leadership, Organizational change, Downsizing, organization, Francophone sub-Saharan Africa

\section{Introduction}

Présentation du contexte, de la justification du sujet, et de la problématique

Les organisations internationales doivent, dans le contexte de la mondialisation s'adapter aux besoins et aux exigences de leurs environnements socio-culturel, économique et politique. Ils doivent faire face à des changements. Un regard rétrospectif sur l'évolution de la théorie des organisations montre que l'attitude des praticiens et théoriciens de la gestion a évolué, mettant plus en vue la problématique du changement en tant que préoccupation dans la réflexion académique sur les stratégies de développement et de performance. La question du changement est ainsi 
devenue une question centrale au management et au leadership des organisations. Le besoin d'un leadership et un management adaptés à ce nouveau contexte surgit de ce fait, et se pose en termes d'intégration de l'environnement économique et professionnel. Ce besoin se présente avec plus d'acuité pour les organisations évoluant sur le contexte africain qui demeure encore sous le poids de beaucoup de pesanteurs socio-culturelles. Les organisations à missions globales sont donc plus éprouvées. le plus grand défi de leur leadership est de s'encastrer dans le dispositif socio-culturel de la localité où elles s'établissent. Ces organisations sont nombreuses sur le continent africain, avec des employés majoritairement locaux dont la gestion constitue le défi susmentionné des directions.

Des raisons objectives, qui sont essentiellement humanitaires et commerciales justifient l'inondation de l'Afrique par les organisations et entreprises internationales. Pendant qu'on observe, d'une part une affluence des organisations internationales non gouvernementales justifiée par les crises humanitaires qui ne cessent de naitre sur le continent, d'autre part, dans les pays relativement stables de ce même continent s'observe un rallye des multinationales. Les matières premières dont regorge ce continent, mais aussi le grand marché qu'il constitue (plus de 2 milliards de population), justifient ces compétitions. L'indispensable nécessité d'intégration de ces organisations dans le nouvel environnement socio-culturel les oblige à procéder à des transformations structurelles et culturelles qui se révèlent de rudes épreuves pour leurs dirigeants et employés.

Il se dégage de ce qui précède une problématique de gestion managériale et de leadership adaptés au contexte africain. Les considérations globales sur les particularités d'un "leadership à l'africaine" ne manquent pas, même si les tentatives pour les conceptualiser existent n'ont toujours pas abouti. Elles souffrent essentiellement d'insuffisance d'évidences empiriques (Patrick Bakengela Shamba, 2007). Les modèles les plus en vue sont, celui du « management africain » tel que proposé par Hernandez (Hernandez, 1997), et celui du «modèle circulatoire de management africain » de Evalde Mutabazi (Mutabazi, 2005). L'intérêt de ces études est justifié par les difficultés à gérer les entreprises africaines selon les méthodes de gestion occidentales qui sont inadaptées aux spécificités de la culture africaine (Hernandez, 1997) mais aussi par des difficultés d'adaptation des dirigeants expatriés en mission en Afrique.

Ce travail de recherche qui porte sur la question contemporaine du changement que vivent les organisations internationales qui évoluent dans le contexte socioculturel spécifique de l'Afrique subsaharienne francophone a pris en compte les sensibilités socioculturelles du cadre géographique, le contexte organisationnel, les acteurs, et leurs interactions. Il s'agit de comprendre, à travers une étude de cas réels, la perspective des différents 
groupes d'acteurs dans de contextes de changement et de dégager le style de leadership qu'il conviendrait d'adopter pour amoindrir les impacts du changement sur les personnes impliquées. Il s'agit d'élucider, à partir de l'étude des cas particuliers de gestion de réduction d'effectifs à OCHA Côte d'Ivoire et à CRISAT Niger, l'impact des sensibilités culturelles sur le processus de conduite des changements et sur leurs finalités. Elle est une réaction aux déficits relatifs aux études empiriques sur le leadership en relation avec la culture (Yammarino F., et al., 2005). Tout en visant la réduction du grand retard des études empiriques sur le contexte spécifique de l'Afrique subsaharienne, elle vise aussi à répondre, modestement, au défi lancé par des chercheurs (Antonakis J., \& House R., 2002) sur la provision de manière empirique de la preuve que les leaders transformationnels peuvent impacter à la fois les hommes et les organisations. Elle a entrepris, à travers l'exploration des différentes étapes des deux processus de réduction d'effectifs, l'examen de leurs impacts sur les employés, ainsi que des styles de leadership de leurs dirigeants, de relever les facteurs qui ont sous-tendu leurs réussites ou échecs du point de vue managérial.

La question initiale de cette recherche est : «Quel impact la culture peut avoir sur les styles de leadership et les processus de réduction d'effectifs dans les organisations internationales en contexte africain subsaharien francophone ?». Les questions subsidiaires auxquelles la recherche fournira des éléments de réponse à son terme sont : 1) Quelles sont les raisons de changements organisationnels à OCHA et ICRISAT ? 2) Quels sont les aspects de gestion des processus de réduction d'effectifs d'OCHA et d'ICRISAT qui sont déterminants pour leurs succès ? 3) Le type de leadership adopté par la direction d'un processus de changement influence-t-il le résultat du processus ? 4) Quelles sont les caractéristiques des types de leadership manifestés par les fonctionnaires qui ont piloté les processus de réductions d'effectifs à OCHA et ICRISAT ? 5) Faudrait-il un modèle de leadership particulier pour réussir les changements dans les organisations internationales en Afrique subsaharienne francophone?

Prenant appui sur les travaux de Hofstede $(1980,19912001)$ sur la culture, les études de Robert House (House et al. 2004) sur la culture et le leadership, les travaux de Bass (1975) sur le Leadership Transformationnel, et ceux de Greenleaf (1977) sur le leadership Serviteur, ainsi que ceux de Hersey et Blanchard (1982), sur le Leadership Situationnel, le postulat de cette recherche qui se situe dans un cadre épistémologique multi paradigmatique combinant les conclusions des études précitées, est que la réussite d'un de processus de changement dans le contexte des organisations internationales en l'Afrique subsaharienne francophone est largement dépendant de la compétence interculturelle des leaders du processus. Le leadership étant un construit qui s'adapte et se réajuste en fonction des enjeux du contexte, une maitrise 
suffisante des spécificités culturelles de la localité serait le premier gage de réussite du processus de changement organisationnel.

\section{Méthodologie}

La présomption que le leadership ne peut être efficace que lorsqu'il intègre largement les spécifications culturelles et les traditions managériales de son contexte a fait choisir le paradigme constructiviste, sur lequel est basé le courant interculturaliste du management, et le paradigme empiriste, qui préconise que la connaissance provient de l'expérience sensible, pour cette étude. En adoptant la posture constructiviste, l'étude entend montrer comment le leadership se construit, se vit, s'ajuste et se réajuste dans le contexte incertain des changements. En adéquation avec les perspectives constructivistes et empiristes, selon les cas, des méthodes hypothéticodéductives et holistico-inductive, et des méthodes quantitatives et qualitatives de recherche ont été utilisées.

Des données, aussi bien primaires que secondaires, ont été recherchées et analysées. Il est particulièrement difficile de situer l'approche de cette étude dans un cadre théorique bien délimité comme c'est généralement le cas dans les recherches en sciences sociales. La grande difficulté de sa réalisation réside dans l'identification d'un cadre de référence théorique qui soutienne la dynamique des interdépendances entre ses concepts (changement organisationnel, leadership, et culture), et qui aide à la sélection, au traitement et à l'analyse des données recueillies, à l'interprétation des événements étudiés, et à la validation des résultats. Sa démarche ne s'inscrivant dans aucun couloir disciplinaire spécifique, il est plus approprié de parler « des théories » plutôt que de risquer de la situer dans un seul cadre théorique. Un cadre épistémologique hétérogène ou multi paradigme composé du constructivisme et de l'empirisme a été retenu pour l'étude. Le caractère multidisciplinaire de la problématique a imposé ce cadre. De toutes les approches de la Sociologie des organisations, les théories des relations humaines (une approche socio psychologique) et de la contingence (approches du management) ont été jugées plus adaptées pour cadrer cette étude dont le choix du sujet a été inspiré par l'expérience personnelle de l'un des auteurs dans une organisation internationale. Elle donne l'opportunité d'analyser scientifiquement, mais aussi de partager l'expérience de cet auteur qui a été le principal agent de mise en œuvre d'un des processus considérés. Le souci d'élargir la population d'étude, mais aussi de diversifier les prismes de lecture, et de couvrir le plus possible le très vaste terrain d'étude (l'Afrique subsaharienne francophone) a orienté dans le choix du second cas qui est celui de la restructuration d'ICRISAT Niger. Le choix d'ICRISAT Niger permet à la fois d'élargir le champ d'étude et de diversifier les perspectives d'analyse. A la différence 
d'OCHA qui est une organisation onusienne, ICRISAT est une Organisation Non-Gouvernementale.

Le recueil des données nécessaires à l'évaluation du processus a été fait avec trois questionnaires: Un questionnaire constitué de dix-sept (17) items regroupés sous quatre (4) dimensions que les auteurs estiment essentielles pour une évaluation des aspects de la planification, du support, de la communication, et du leadership des processus étudiés ; Un questionnaire constitué des 18 items (qualités) regroupés autour des 6 dimensions du leadership orienté humain que l'étude de GLOBE a attribué à cette zone ; Un questionnaire adapté du modèle du collaborateur le moins apprécié (CMA) tel que conçu par Fiedler (1967) pour rechercher l'adéquation entre les leaders et les situations.

\section{Résultats et Discussions}

\section{A. Les causes des réductions d'effectifs d'OCHA CI et ICRISAT Niger}

Il se dégage de l'analyse faite que, bien que les diverses perspectives étudiées par les chercheurs indiquent que beaucoup de stratégies de changement existent pour les organisations, les contraintes économiques, les compulsions institutionnelles, les croyances idéologiques et les perspectives irrationnelles pourraient constituer quelques-unes des causes de restructuration ; il est moins évident que ces causes s'excluent mutuellement ; ainsi, une décision de réduire les effectifs pourrait être motivée par plusieurs impératifs, dépendant des contextes. Les deux cas de réduction d'effectifs considérés ne font pas exception: la réduction d'OCHA satisfait à des exigences institutionnelles et stratégiques, tout en répondant à des contraintes économiques; celle d'ICRISAT répond par contre à des contraintes purement économiques.

Les tests de validation des quatre hypothèses relatives à la planification et la mise en œuvre du processus des réductions d'effectifs réalisées suivant les règles établies ont permis d'obtenir les résultats suivants : les aspects des processus de réductions d'effectifs à OCHA et ICRISAT qui sont déterminants pour la réussite sont : la planification du processus ; le soutien apporté aux personnes impliquées ; la communication dans le processus ; le leadership manifesté. Surtout une stratégie adéquate de communication et un excellent leadership peuvent permettre de juguler les problèmes qui découleraient des deux premières dimensions.

\section{B. L'orientation relation - tâche dans les styles de leadership des deux processus}

La cinquième hypothèse nulle porte sur le leadership qui est la quatrième dimension de l'évaluation du processus de gestion de réduction d'effectifs. Elle dit que «Le leadership manifesté dans le processus de 
réduction d'effectifs d'OCHA et d'ICRISAT n'était pas satisfaisant à cause du manque d'attention sur les personnes concernées ». Les indicateurs retenus de l'évaluation de cette dimension sont: 1) partage de la vision du changement ; 2) l'attitude du management par rapport au personnel impacté ; 3) les préoccupations des employés avaient été traitées ; 4) l'étendue des actions de support au bénéfice des employés impactés. Les résultats de l'enquête entreprise à ICRISAT indiquent que les personnes impactées ne sont globalement pas satisfaites du leadership manifesté par les exécutants. Bien qu'ils trouvent acceptable l'attitude des exécutants (moyenne de réponse de $2,86)$, ils estiment que la vision du changement n'avait pas été partagée avec eux, leurs préoccupations n'avaient pas été considérées, et que les activités de support n'étaient pas du tout suffisantes (une moyenne de 1,75). Comme conséquence de cette évaluation peu satisfaisante de la dimension leadership, 1'hypothèse nulle a et été acceptée et son alternative rejetée. Le leadership dans le processus de réduction des effectifs à ICRISAT n'était donc pas satisfaisant.

Le résultat de l'évaluation de la même dimension à OCHA parait plus luisant. Les quatre indicateurs retenus avaient été évalués positivement de sorte qu'il a été conclu le rejet de l'hypothèse nulle et l'acceptation de sa variante positive. Le leadership dans le processus de réduction des effectifs à OCHA était donc satisfaisant. Comme pour la dimension communication, la précipitation du processus est à incriminer pour cette mauvaise évaluation.

Le rôle du leadership dans un processus de changement est dévolu à la haute direction de l'organisation qui a décidé de la nécessité du changement, et qui en donne les orientations. Le succès du processus dépend donc très étroitement des choix de leviers fait par le leadership pour mitiger les incontournables impacts sur les employés et les structures organisationnelles. En sus de veiller à la conformité de la déclinaison du dispositif, il doit être à même de fournir une vision globale, une stratégie, une direction comme signe de visibilité du devenir du destin collectif (Cascio, 2009; Iverson \& Zatzick, 2011; K. E. Mishra, et al., 1998).

Il transparait des résultats de l'enquête faite à ICRISAT sur cette dimension importante que malgré la bonne attitude des dirigeants reconnu par les personnes impactées, le temps imparti au processus ne leur permettait pas de faire usage des leviers à leur disposition pour amoindrir les divers impacts qui justifient l'amertume exprimée par les ex-employés et les survivants. Les approches de leadership adoptés par les exécutants des deux processus étant globalement les mêmes (leadership mixte avec une forte orientation humaine), les employés étant majoritairement de mêmes traditions culturelles, la différence de perception ne peut que difficilement être attribuée à d'autres aspects que le temps de manœuvre.

Deux autres éléments qui auraient aussi contribué au grand choc ressenti par les employés impactés par le changement à ICRISAT sont leurs 
âges avancés et leurs longues années de loyaux services à l'organisation. Onze (11) des dix-sept (17) personnes séparées qui ont participé à l'enquête ont plus de 46, avec une moyenne de vingt-trois (23) années de service à ICRISAT. A l'inverse, la population impactée à OCHA est plus jeune $(80 \%$ des survivants et $50 \%$ des personnes séparées ont moins de 45 ans), plus diversifiée (africains et non africains) avec peu d'années aux Nations Unies (les personnes séparées ayant participé à l'enquête ont environ 5 années dans l'organisation et les survivants en ont 9,5). Avec une espérance de vie de 56 ans pour les hommes et 59 ans pour les femmes ${ }^{6}$, cela est une évidence qu'à la quarantaine, il y a peu d'espoir pour un changement de carrière, d'où le grand choc de ces personnes impactées. Le manque d'accompagnement psychologique nécessaire dans cette situation en a rajouté au ressentiment exprimé.

Ces observations sur l'importance de la dimension leadership corroborent beaucoup d'autres travaux qui ont été consacrés à la gestion de changement. Comme 1'a dit Kotter (2015), « une transformation réussie vient de 70 à $90 \%$ de leadership et de 10 à $30 \%$ de management ». C'est par lui que sont déployés tous les autres éléments (la communication, le soutien etc.) qui contribuent au succès du processus. Il est lié à la motivation, au comportement interpersonnel et au processus de communication. Il est important dans sa tentative de réduction de la démotivation. Avant de passer aux observations sur les résultats relatifs aux deux dernières questions de notre recherche, il importe de faire un point général sur l'évaluation des quatre dimensions qui constitue le processus de mise en œuvre des réductions d'effectifs dans les deux organisations.

Les conclusions de l'analyse des données biographiques des participants d'OCHA montrent que les exécutants constituent le groupe le plus âgé et le plus expérimenté, les survivants sont plus jeunes mais plus expérimentés et que les personnes concernées qui, eux, sont plus âgées que les survivants, mais moins anciens dans l'organisation; ces harmonies sont une preuve que la sélection n'était pas basée sur des critères subjectifs. OCHA, en tant qu'entité des Nations Unies, a suivi les articles 9.6 (e) et 13.1 (d) du Statut du personnel qui présentent les critères de conservation, et a organisé des tests compétitifs quand nécessaire. ICRISAT, une organisation à vocation de recherche agronomique, a opté, pour pouvoir garder sa capacité opérationnelle et continuer sa mission, se séparer d'abord du personnel de soutien. Le volet de l'identification des agents de la recherche à licencier a été laissé aux directeurs de programmes comme le dit le procès-verbal de la première réunion d'information sur le changement.

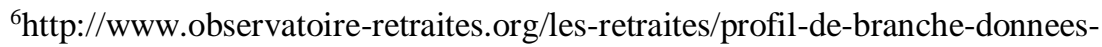
effectifsemplois/les-retraites-en-afrique/ consulte le 14 avril 2019.
} 
Il faut noter qu'une fois la décision de réduire des effectifs a été prise, beaucoup de critères existent pour déterminer qui licencier et qui laisser (Cascio, 2009). En règle générale, les employeurs sont libres d'utiliser les critères de leur choix pour licencier leurs employés, s'ils ne veulent pas faire de discriminations ou du favoritisme.

Les conclusions de l'analyse des données biographiques des participants d'ICRISAT montrent que les personnes concernées constituent l'un des groupes les plus âgés (77\% ont plus de 46 ans) et a une moyenne de nombre d'années d'expérience (23 ans) supérieure à la moyenne générale d'années d'expérience des répondants (19 ans). Les survivants comptent parmi eux beaucoup de personnes âgées de plus de 46 ans (65\%), mais aussi une bonne proportion d'employés (35\%) dont l'âge varie entre 26 et 45 ans. Les exécutants ont par contre tous plus de 46 ans même si leur moyenne d'années d'expérience est égale à celle des survivants (17) et inferieur à celle des personnes concernées ( 23 ans).

Avoir des exécutants matures et expérimentés est l'idéal pour la gestion de tels processus qui impactent profondément des vies et ont beaucoup d'implications légales. Le fait qu'une bonne proportion des survivants soit jeune et que la plupart des employés très âgés soit licencié est conforme à de nombreux principes de gestion des ressources humaines appliqués dans de tels processus. Ces harmonies sont une preuve que la sélection n'était pas basée sur des critères subjectifs.

Il convient cependant de noter que, bien que cette configuration de personnes impliquées dans le processus soit bénéfique pour la continuité de l'organisation, elle augmente l'impact social négatif de la perte d'emploi. Les problèmes de santé critiques qui ont été gérés de manière concomitante avec le processus et dont il est fait écho dans la section sur les impacts sont une conséquence de cette situation. Il n'existe aucun doute sur l'existence d'un effet « licenciement » sur la santé des personnes, d'autant plus que leurs récits donnent des détails sur la réalité de l'enchaînement «licenciement symptômes - prescription médicale ». Le désarroi ressenti et décrit par nombre de personnes concernées montre que le choc est rude et provoque, pour une partie d'entre eux, une altération de la santé.

La priorité pour le fonctionnaire qui perd son emploi étant de trouver un nouvel emploi, toute aide pour l'obtenir devrait avoir la plus grande valeur aux yeux des victimes et devrait être déterminante dans leur évaluation du processus. Mais ce ne fut apparemment pas le cas à ICRISAT, comme le dit cet enquêté :

"Le paquet n'était pas consistant pour des agents ayant passé plusieurs années à l'ICRISAT. L'ICRISAT devait aider les concernés à trouver du travail parce qu'il est difficile pour eux d'en trouver, vu l'âge avancé pour la plupart d'entre eux». 
Supposant que les deux processus se sont déroulés dans de contextes socio-culturels identiques, avec exécutants imbus de mêmes valeurs culturelles et dotés de mêmes principes managériaux, les auteurs ont fait une compilation des données recueillies. L'analyse faite de ces données indique que la perception des participants des 2 processus réunis sur les dimensions planification et soutien est la même : négative. Les perceptions globales sur les dimensions communication et leadership sont par contre positives quand elles sont mises ensemble. Ces résultats laissent croire qu'avec un peu plus de communication et de leadership la tendance à ICRISAT peut être aisément renversée, et partant le résultat de l'évaluation de tout le processus.

En conclusion à cette partie, et comme réponse à la deuxième question de recherche, il peut être affirmé que les aspects des processus de réductions d'effectifs à OCHA et ICRISAT qui sont déterminants pour la réussite sont : la planification du processus ; le soutien apporte aux personnes impliquées ; la communication dans le processus; le leadership manifesté. Surtout une bonne stratégie de communication et un bon leadership peuvent permettre de juguler les problèmes qui découleraient des deux premières dimensions.

\section{La dimension relation humaine dans le leadership manifesté dans les deux processus}

La sixième hypothèse nulle de la recherche dit que « La réussite de conduite de changement en Afrique subsaharienne francophone ne nécessite pas un leadership dominé par les rapports humains» Il se dégage de l'analyse des données recueillies par le moyen du questionnaire LPA qui vise à évaluer les styles de leadership adoptés par les différentes personnes qui ont eu la responsabilité de gérer les deux processus soumis à étude que la moitié de ces personnes (3 sur 6) avaient adopté une approche qui privilégie à la fois les relations humaines et les tâches à accomplir ; des 4 exécutants du processus d'ICRISAT, le premier responsable (le Directeur régional) est perçu comme quelqu'un qui a une approche fortement relationnelle ; le responsable des ressources humaines a une approche perçue comme mixte ; tandis que les approches des du Représentant pays et de l'Administrateur régional sont perçues comme privilégiant plus les tâches à accomplir que les relations avec les personnes. La moyenne de performance des quatre exécutants est de 791. 791 étant très proche de la limite minimale du leadership mixte, l'inclinaison du style de leadership vers les taches est bien faible.

Les deux exécutants du processus d'OCHA avaient un style perçu comme recherchant de bons rapports avec les fonctionnaires, sans perdre de vue le fait que les tâches doivent être accomplies. Ils ont une moyenne de scores de 849 qui indique bien un leadership mixte.

Supposant encore une homogénéité culturelle des deux contextes, les auteurs ont compilé les moyennes des exécutants des deux processus. La 
moyenne obtenue de 810 indique que globalement le leadership manifesté dans les deux processus est mixte.

Le processus de réduction d'effectifs d'OCHA paraissant le plus satisfaisant au regard des résultats de l'évaluation du processus (au chapitre 3), la vérification de l'hypothèse 6 a été axé sur le résultat de l'évaluation des exécutants du processus d'OCHA. La règle de vérification de cette hypothèse était de rejeter l'hypothèse nulle et d'accepter sa variante si la moyenne des scores des exécutants du processus évalué comme satisfaisant est comprise entre 792 et 917 ou supérieur à 918. La moyenne des scores des exécutants d'OCHA étant de 849, qui se situe bien entre 792 et 917, par voie de conséquence, l'hypothèse nulle : « la réussite de conduite de changement en Afrique subsaharienne francophone ne nécessite pas un leadership mixte ou dominé par les taches » a été rejetée et son alternative qui dit que «La réussite de conduite de changement en Afrique subsaharienne francophone nécessite un leadership mixte ou dominé par les relations humaines » a été acceptée.

Il peut être observé de l'analyse des résultats qu'un style de leadership mixte, avec une forte inclinaison relationnelle (moyenne de 849) est attaché au processus qui a été évalué comme un succès (le processus d'OCHA), tandis qu'au processus évalué comme pas satisfaisant (processus d'ICRISAT) est associé un style de leadership orienté vers les taches. Il peut être déduit, comme réponse à la troisième question de recherche, que le type de leadership adopté par la direction d'un processus de changement influence bien le résultat $\mathrm{du}$ processus. En l'occurrence l'inclinaison relationnelle du leadership des exécutants des deux processus a été instrumentale dans l'atténuation des impacts humains.

Ce résultat corrobore en outre les conclusions de l'étude d'Evralde Mutabazi (2005) qui ont abouti à sa théorisation du modèle circulatoire. En effet, les caractéristiques principales de ce modèle sont l'approche intégrative, la relationalité, le développement de la communauté, et la considération relative du temps. Pour ce modèle, il est important pour l'homme de s'intégrer dans la nature, de veiller permanemment à l'amélioration de sa relation avec la nature et les membres de sa communauté.

Il faut relever cependant que l'orientation vers les taches et l'orientation vers les personnes constituent deux options qui ne s'excluent pas l'une l'autre. Si chacune d'elle restait exclusive, cela conduirait l'organisation dans une situation ou les hommes seraient vus comme des robots (seulement leur labeur compterait, comme dans l'école classique du management incarnée par le français Henry FAYOL, l'américain Frederick TAYLOR, et l'allemand Max WEBER), ou, à l'opposé, une situation où tout serait fait pour plaire aux hommes, et le mandat et les objectifs de l'organisation seraient délaissés. L'approche conciliante de ces deux options a été proposée par Elton Mayo (1933) qui, en réaction au taylorisme affirme le rôle essentiel du facteur 
humain et l'importance des relations dans la détermination de la productivité économique. Cette approche réintroduit ainsi les facteurs psycho-sociaux au cœur de l'analyse de l'organisation, laissant le champ ouvert à l'application des méthodes issues de l'analyse sociologique. À partir de cette analyse, des auteurs comme Abraham Maslow (1943) ou Frederick Herzberg (1959) ont approfondi l'étude de la motivation, cet ingrédient qui est essentiel au succès dans les organisations. Un leader motivateur inspire ses subordonnés à devenir performants et à considérer l'intérêt, à long terme, de l'organisation au détriment de leur propre intérêt, de court terme : substance de la théorie du leadership transformationnel de Bernard Bass (1985, 1990). Pour Bernard Bass (1985; 1990), le focus du leader doit être sur la croissance de l'organisation de sorte que son comportement favorise l'engagement des employés pour l'accomplissement des objectifs de l'organisation par le biais de leur autonomisation (Yukl, 1998). Les caractéristiques principales de cette théorie de leadership sont le charisme, l'inspiration, la stimulation intellectuelle et la considération. Le leader transformationnel motive les employés à des niveaux plus élevés de performance pour le bien de l'organisation (Burns, 1998; Yukl, 1998). Il en résulte alors une performance améliorée de l'employé. (Burns, 1998; Yuk1, 1998). Il transparait donc un lien entre le développement de l'organisation et celui des employés ; ce lien rapproche le leadership transformationnel du style de leadership mixte qui parait adapté pour un contexte de changement. Il permet le développement de l'organisation en même temps que celui de l'employé qui s'accompli par son travail.

La septième et dernière hypothèse nulle de la recherche dit que « Le style de leadership à dominante humaine n'est pas gage de réussite de conduite de changement dans les organisations internationales implantées en Afrique subsaharien francophone »

Il se dégage de l'analyse des données recueillies par le moyen du questionnaire adapté des conclusions de la recherche de GLOBE qui vise à évaluer l'orientation humaine dans le style de leadership adopté par les différentes personnes qui ont eu la responsabilité de gérer les deux processus soumis à étude que la dimension de leadership humain est la plus fortement manifestée (une moyenne de 4.0) par les leaders de ces processus, suivie, par ordre d'importance, de l'esprit d'équipe $(3,8)$, du charisme $(3,7)$, et du leadership participatif $(3,7)$. Il transparait en outre que ces leaders sont très auto-protecteurs $(3,3)$ et peu autonomes $(2,9)$. 
Tableau 1 : Comparaison du résultat de notre étude sur la base de la théorie de GLOBE avec les conclusions initiales de GLOBE.

\begin{tabular}{|c|c|c|}
\hline \multicolumn{2}{|c|}{$\begin{array}{l}\text { Conclusions de GLOBE concernant la zone de } \\
\text { l'Afrique subsaharienne (Adapted from House, } \\
\text { et al. (2004). }\end{array}$} & $\begin{array}{l}\text { Resultat de notre recherche sur la base des } \\
\text { conclusions de GLOBE }\end{array}$ \\
\hline \multirow{6}{*}{ 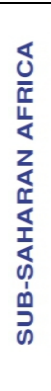 } & Humane-Oriented Leadership & Score \% \\
\hline & Charismatic/Nalue-Based Leadership & \\
\hline & Team-Oriented Leadership & Leadership charismatique \\
\hline & Participative Leadership & Leadership orienté par l'equipe \\
\hline & Self-Protective Leadership & Leadership Autonome \\
\hline & Autonomous Leadershipl & Leadership Auto-protecteur $3.3 \%$ \\
\hline
\end{tabular}

Source : conception des auteurs de l'article (Dounia BERE et Patrick ADON).

Sur cette figure peut s'observer à gauche les dimensions du leadership culturel tels que présentées par l'étude initiale GLOBE, et a droit les mêmes dimensions telles que issues de l'étude empirique. La dominance de la dimension de l'orientation humaine sur les deux graphiques est la preuve la plus évidente que les résultats de notre étude corroborent bien les conclusions de l'étude GLOBE. La légère avance de la dimension du leadership autoprotecteur du résultat de la présente étude sur celle de GLOBE se justifierait certainement par le fait que les gestionnaires des deux processus étudiés ne sont que des relais de leurs hautes directions basées dans leurs sièges en Occident; ils ne sont pas entièrement libres décideurs des options qu'ils défendent, donc pas totalement autonomes.

Ces résultats permettent, conformément à la règle établie (rejet de l'hypothèse si la moyenne de scores de la dimension de leadership humaine est égale ou supérieure à 3 ) de rejeter la septième hypothèse nulle, «le style de leadership à dominante humaine n'est pas gage de réussite de conduite de changement dans les organisations internationales implantées en Afrique subsaharien francophone », et d'accepter son alternative qui dit que « le style de leadership à dominante humaine est gage de réussite de conduite de changement dans les organisations internationales implantées en Afrique subsaharien francophone ».

\section{Synthèse des conclusions et proposition d'un modèle}

\section{A. Synthèse des conclusions}

\section{Les conclusions de GLOBE}

Des conclusions de Hofstede (1980) et de l'étude de GLOBE que ces dernières ont inspiré par la suite sur les différenciations culturelles, ainsi que des conclusions des études de Marcel Zadi (1998), d'Evalde Mutabazi (2001), 
etc., il se dégage un portrait culturel de l'Afrique subsaharienne caractérisé principalement par une forte orientation humaine. L'observation de cette culture au contact avec le management a révélé les spécificités que l'étude de GLOBE a mises en exergue, et dont la pertinence a été, une fois de plus, confirmée par les résultats de la présente recherche empirique. Il est donc important de tenir compte de ces conclusions dans la pratique du leadership dans le contexte de l'Afrique Subsaharienne francophone.

$\mathrm{Au}$ regard des similitudes de caractéristiques du leadership humain (conclusions de GLOBE) avec les théories du leadership transformationnel de Bernard Bass (1985, 1990), et du leadership serviteur de Greenleaf, qui sont également deux théories très utilisées en contexte de changement, il peut être conclu qu'un modèle de leadership issue d'une mixture de leurs caractéristiques serait idéal pour la gestion de changements organisationnels en contexte de l'Afrique subsaharienne francophone.

En effet, les conclusions de l'étude de GLOBE disent que le leader idéal pour l'Afrique sub-saharienne doit être fortement orienté humain, être modeste, compatissant, et sensible aux préoccupations de ses suiveurs ; il doit être relativement charismatique, motivé par des valeurs de la dignité humaine, avoir un esprit d'équipe, être participatif, et auto-protecteur. Le leader qui agit seul est considéré comme pas performant dans ces pays. En bref, il doit être attentionné, source d'inspiration, collaboratif, et pas égocentrique.

Le Leadership orienté humain est un style de leadership qui met l'accent sur le fait d'être serviable, prévenant, compatissant et généreux. Ce type de leadership comprend la modestie et la sensibilité aux autres. Les idées caractéristiques de ce leadership se trouvent bien condensées dans les termes Humilité et Empathie. L'humilité est le sentiment qu'une personne éprouve de sa faiblesse, de son insuffisance, et qui la pousse à s'abaisser volontairement en réprimant son orgueil (Dictionnaire Le Grand Robert). Il est un synonyme de «modestie », avec qui il est interchangeable dans beaucoup d'usages.

\section{Le leadership serviteur et la gestion des changements}

Très peu de modèles alternatifs et innovants de leadership qui existent pourraient apporter de meilleures réponses aux problèmes de management des hommes et des organisations en général et dans le contexte de gestion de changement en particulier. Du rang des rares paradigmes alternatifs qui existent se compte le «servant leadership » ou le leadership serviteur, d'origine américaine, qui est présenté dans cette étude. Daniel Bellet (2013) le présente comme « un paradigme puissant et humaniste pour remédier à la crise du management $»$.

Il s'agit d'un modèle de management des hommes et des organisations qui offre de nouvelles réponses opérationnelles efficaces à de nombreux problèmes managériaux et de « dysfonctionnements» auxquels sont 
confrontées beaucoup d'organisations, et surtout lorsqu'elles sont en restructuration. Il représente une piste particulièrement intéressante pour gérer les changements en Afrique subsaharienne où les considérations humaines sont dominantes et très sensibles. Les perspectives qu'il offre permettent de construire un nouveau paradigme de leadership et un management humain, capables de concilier les performances organisationnelles et le bien - être au travail des employés.

Le modèle du «servant leadership » considère que les employés sont la fin et non de simples moyens, et qu'à ce titre, ils doivent être mis effectivement au cœur du management puisque c'est eux qui sont la principale source de création de valeur (Bellet, 2013). Dans cette perspective, le leader en contexte de changement doit prioritairement aider les employés impactés, en usant de tous les leviers à sa disposition, afin de réduire au minimum possible les effets du changement dans tous les aspects de leur vie.

\section{Le leadership transformationnel et la gestion des changements}

Bernard Bass $(1985,1990)$ qui a développé l'approche du leadership transformationnel, sur la base des travaux de J. M. Burns (1978), a identifié quatre des aspects principaux de cette théorie qui constituent sa base philosophique. Ce sont le CHARISME, l'INSPIRATION, la STIMULATION INTELLECTUELLE et LA CONSIDERATION. Un leader qui fait preuve de ces qualités inspire ses subordonnés à devenir performants et à considérer l'intérêt, à long terme, de l'organisation au détriment de leur propre intérêt, de court terme. Son bon usage permet, au-delà des comportements, à modifier la perception et les croyances de gens qu'on dirige ; c'est ce qui justifie son efficacité dans les environnements de turbulence et d'adaptation continue. Il repose sur quatre composantes principales.

La première est le charisme idéalisé : Les subordonnés admirent, respectent et font confiance à leur leader. Le leader transformationnel est donc perçu comme un modèle aux yeux de ses subordonnés qui veulent lui ressembler. Cette idéalisation du leader s'explique par l'intégrité et la moralité de sa conduite; il agit selon un système de valeurs existentielles qui ne peuvent être négociées.

Ensuite, il y a la motivation par stimulation: Le leader transformationnel procure un sens au travail et des défis à ses subordonnés, ce qui les motive à performer ; il communique une vision positive de l'avenir, explicite ses attentes et se montre engagé envers la vision et les objectifs de l'organisation; il fait preuve d'enthousiasme et d'optimisme, ce qui favorise l'esprit d'équipe.

La troisième est la stimulation intellectuelle : Le leader transformationnel incite ses subordonnés à être créatifs et à innover en questionnant les hypothèses, en recadrant les problèmes et en envisageant les 
situations passées sous des angles nouveaux. Les subordonnés sont impliqués dans le processus de résolution des problèmes et ne sont pas blâmés ou critiqués publiquement pour leurs erreurs.

La quatrième composante est la considération individuelle : le leader prend le temps de connaître à minima les attentes et situations de chacun de ses collaborateurs. Il prodigue des conseils personnalisés et met l'accent sur ce qu'il apprécie particulièrement dans le travail de chacun. Il trouve le temps dans son agenda de faire un travail en face à face pour soutenir individuellement la progression de ses collaborateurs. Il permet à ses subordonnés de se développer et de grandir professionnellement.

Tableau 2 : Résumé des caractéristiques des trois théories contribuant au modèle de leadership souhaité pour le contexte étudié.

\begin{tabular}{|c|c|c|c|}
\hline $\begin{array}{l}\text { Caractéristiques du } \\
\text { Leadership orienté } \\
\text { Humain selon de l'Afrique } \\
\text { subsaharienne - selon } \\
\text { GLOBE }\end{array}$ & $\begin{array}{l}\text { Caractéristiques du } \\
\text { Leadership } \\
\text { Transformationnel- } \\
\text { Bernard Bass. }\end{array}$ & $\begin{array}{l}\text { Caractéristiques du } \\
\text { Leadership Serviteur } \\
\text { - Greenleaf. }\end{array}$ & $\begin{array}{l}\text { Caractéristiques du } \\
\text { modèle idéal pour le } \\
\text { contexte de } \\
\text { changement en Afrique } \\
\text { subsaharienne. }\end{array}$ \\
\hline $\begin{array}{l}\text { 1.Être fortement oriente } \\
\text { humain (Serviable, } \\
\text { Prévenant, Compatissant } \\
\text { Généreux, Modeste } \\
\text { La sensibilité aux } \\
\text { personnes qu'on dirige) } \\
\text { 2.Avoir du charisme } \\
\text { 3.Avoir un esprit d'équipe } \\
\text { 4.Être participatif } \\
\text { 5.Être auto-protecteur. } \\
\text { 6.Être autonome }\end{array}$ & $\begin{array}{l}\text { 1. L'influence } \\
\text { charismatique, } \\
\text { 2. L'inspiration, } \\
\text { 3. La stimulation } \\
\text { intellectuelle } \\
\text { 4. La considération } \\
\text { individualisée. }\end{array}$ & $\begin{array}{l}\text { 1. Écoute } \\
\text { 2. Empathie } \\
\text { 3. Guérison } \\
\text { 4. Conscience } \\
\text { 5. Persuasion } \\
\text { 6. Conceptualisation } \\
\text { 7. Prévoyance } \\
\text { 8. Intendance } \\
\text { 9. Engagement à faire } \\
\text { grandir les personnes } \\
\text { 10. Construction de } \\
\text { Communauté }\end{array}$ & $\begin{array}{ll}\text { 1. } & \text { Serviable } \\
\text { 2. } & \text { Modèle } \\
\text { 3. } & \text { Attentionné } \\
\text { 4. } & \text { Réfléchi } \\
\text { 5. } & \text { Travailleur } \\
\text { 6. } & \text { Ecouteur } \\
\text { 7. } & \text { Mentor } \\
\text { 8. } & \text { Persuadeur } \\
\text { 9. } & \text { Allocentrique } \\
\text { 10. } & \text { Thérapeute } \\
\text { 11. } & \text { Honnête } \\
\text { 12. } & \text { Idéaliste } \\
\text { 13. } & \text { Econome }\end{array}$ \\
\hline
\end{tabular}

Source : conception des auteurs de l'article (Dounia BERE et Patrick ADON)

La dominance de la dimension du leadership humain dans le résultat de cette recherche empirique menée pour évaluer les styles de six dirigeants d'organisations internationales dans le contexte de l'Afrique subsaharienne est une preuve supplémentaire de la pertinence des conclusions de l'étude de GLOBE (House et al. 2004). Les conclusions de l'étude de GLOBE qui établissent la relation entre la culture et le leadership conseillent un leadership à forte orientation humaine pour le contexte de l'Afrique subsaharienne. Elles révèlent que les pays varient dans leurs approches du leadership en fonction de leurs tendances culturelles.

Selon l'analyse de GLOBE, un leader idéal de l'Afrique subsaharienne est modeste, compatissant, et sensible à la population. Il doit être relativement charismatique, motivé par des valeurs de la dignité humaine, avoir un esprit d'équipe, être participatif, et auto-protecteur. En bref, il doit 
être serviable, prévenant, compatissant, généreux, attentionné, source d'inspiration, collaboratif, et pas trop égocentrique.

Ces caractéristiques rejoignent en outre les caractéristiques du leadership serviteur de Robert K. Greenleaf (1904-1990) et ses dérivées (Patterson, 2003 ; J. Barbuto et D. Wheeler, 2006). Pour la théorie de Greenleaf, l'intérêt ne devrait pas motiver le leader serviteur qui devrait au contraire montrer un niveau supérieur de motivation (Greenleaf, 1977; Pollard, 1996). La contribution principale de Greenleaf à la compréhension du leadership-serviteur était la délinéation des caractéristiques du leadershipserviteur. Il estime que les leaders serviteurs font preuve principalement de dix comportements qui sont : l'écoute, l'empathie, la guérison, la conscience, la conceptualisation, la persuasion, prévoyance, l'intendance, l'engagement à développer les personnes, et la construction de la communauté.

De la mixture des caractéristiques de ces trois théories nait un modèle qui est l'objet de la section qui suit.

\section{B. Le Modèle de leadership SMART et empreint d'EMPATHIE}

Il n'est pas présenté ici une théorie mais plutôt un modèle de leadership, à partir d'une combinaison des conclusions de l'étude des deux cas considérés. Généralement, un modèle est développé dans le prolongement d'une théorie, dont il est en fait une projection. Parce qu'il fait référence à une gamme plus limitée de situations que la théorie dont il est issu, le modèle est habituellement d'application plus réduite. Le modèle n'est pas un outil explicatif et de généralisation mais il joue un rôle important dans la formulation des théories (Willett, 2012).

L'analyse des caractéristiques spécifiques des trois théories et le portrait culturel réalisé du contexte ont permis de comprendre et d'identifier les éléments requis pour le model de leadership recherché. Ces éléments peuvent aider à avoir une compréhension plus fine et différenciée des pratiques de leadership en contexte de changement en Afrique subsaharienne francophone.

On observe sur le tableau ci-dessus que les trois premières colonnes présentent respectivement 1) les caractéristiques générales du leadership de l'Afrique subsaharienne vues du prisme de l'étude de GLOBE, 2) les caractéristiques du leadership transformationnel qui est réputé plus efficace pour la gestion des changement, 3) et les caractéristiques du leadership serviteur considéré comme le paradigme alternatif pour la gestion des changement dans le contexte sous examen, à cause de sa forte propension humaniste. Dans la quatrième et dernière colonne sont réunies les treize (13) caractéristiques qui résument les éléments des trois précédentes colonnes et qui pourront servir de fondation pour un modèle de leadership spécifique pour la gestion de changement dans les organisations internationales dans le 
contexte de l'Afrique subsaharienne. Les premières lettres des 13 vocables qui caractérisent le modèle de leadership postulé pour l'Afrique subsaharienne mises ensemble comme un acrostiche ${ }^{7}$ donnent deux termes, SMART et EMPATHIE qui, en plus de l'harmonie sémantique avec le concept faciliteraient sa mémorisation.

\section{Un leader SMART}

Le sigle SMART (Spécifique, Mesurable, Acceptable, Réaliste, Temporellement défini) qui est utilisé en marketing pour qualifier des objectifs intelligents est ici en emprunt pour décliner les qualités nécessaires pour un leader gestionnaire du changement organisationnel en Afrique subsaharienne. Il doit être Serviable, Modèle, Attentionné, Réfléchi, et Travailleur.

* Serviable: La serviabilité est tout ce que le leadership transformationnel a de moins que le leadership serviteur; une petite dose de serviabilité dans un leadership transformationnel ne le ferait donc que tendre vers la forme idéale de leadership.

Modèle : Etre modèle signifie vivre les comportements que vous voulez que les autres adoptent. Ceci met le leader sur la première scène. Les gens croient moins ce qu'ils entendent les dirigeants dire ; ils croient plus ce qu'ils les voient constamment faire.

Attentionné : Le leader doit faire attention aux individualités du groupe afin de les valoriser et les développer. C'est en faisant attention aux sensibilités culturelles des hommes qu'il peut tirer le maximum de profit d'eux.

Réfléchi, responsable et relationnel : tout leader transformationnel doit porter la marque de la réflexion et de la responsabilité. Etant le garant de la cohésion du groupe, il se doit d'être en bons termes avec tous les membres.

Travailleur, transformateur: La transformation est la finalité du processus de changement; le leader se doit de travailler ardemment pour y arriver.

\footnotetext{
${ }^{7}$ Un acrostiche est un jeu littéraire qui à consiste à écrire un poème dont on peut lire un mot formé par les initiales des vers. Ce mot est souvent le nom de l'auteur, le nom de celui à qui on dédie le poème ou un mot en rapport avec le titre du poème. En savoir plus sur https://www.laculturegenerale.com/acrostiche-definition-celebres-exemples/ | La culture générale
} 
Figure 1 : L'élément SMART du modèle de leadership

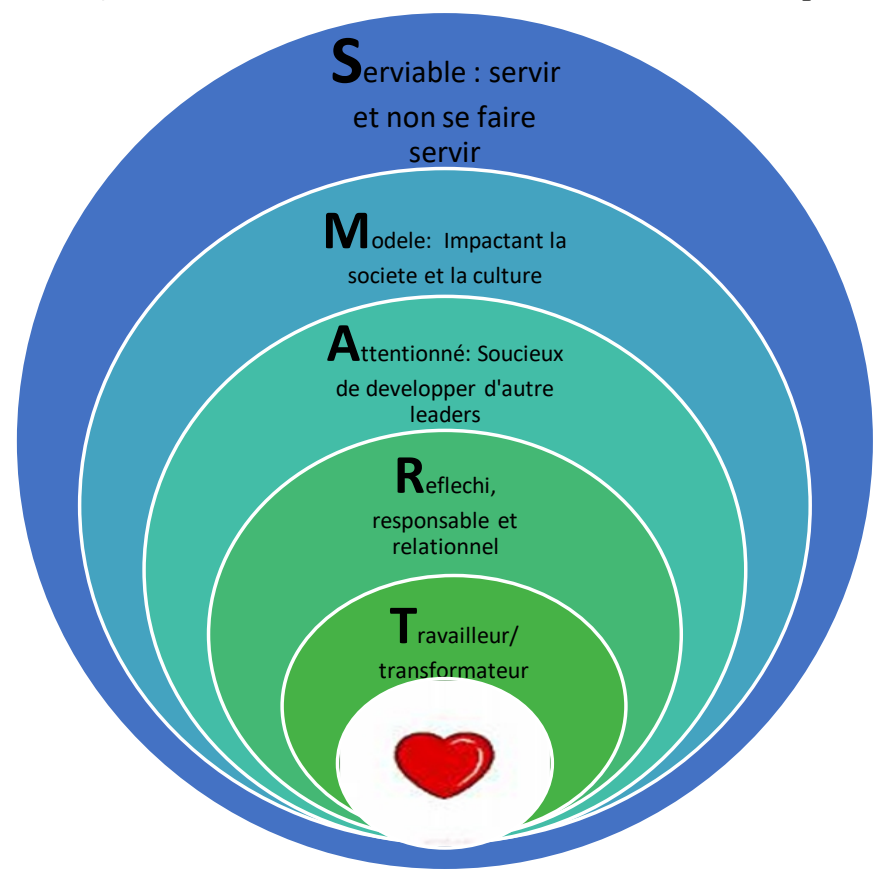

Source : conception des auteurs de l'article (Dounia BERE et Patrick ADON).

\section{Un leader qui a de l'EMPATHIE pour les gens qu'il dirige}

Procédant de la même manière que ci-dessus, les premières lettres des huit derniers termes donnent le terme EMPATHIE qui est une caractéristique commune à toutes les trois théories et donc centrale au nouveau modèle. "L'empathie du leader est une qualité qui donne le goût aux autres de le suivre", souligne M. Norrie, coauteur du livre The A to Z Guide to SoulInspiring Leadership. Grâce à l'empathie, le leader comprend mieux les émotions des gens dans une situation de changement. Et une fois qu'on comprend les gens, on peut apprendre le reste, ajoute-t-il. Dans un environnement complexe, être un leader dans la durée requiert des compétences spécifiques. La diversité des populations à manager, la diffusion de l'information, l'incertitude des changements nécessitent de maîtriser des compétences critiques telles que l'empathie, la résilience, l'assertivité, l'imagination créative, etc. 
Figure 2 : L'élément EMPATHIE du modèle de leadership

-Ecoute: Le leader a un engagement profond à l'écoute attentive des autres.
L'écoute également englobe : contacter sa propre voix intérieure et chercher à
comprendre quelles parties du corps, de l'esprit ou de l'âme communiquent.
L'écoute, associée à des périodes régulières de réflexion, est essentielle à la
croissance du leader-serviteur.
- Mentorat: Le leader-serviteur et profondément engagé dans la croissance de
chaque individu dans son institution.
- Persuasion: un leader-serviteur utilise la persuasion, plutôt que
l'autorité de la position qu'il occupe.
-Allocentrisme: Le leader-serviteur tâche de comprendre et de compatir avec les
autres. Le besoin des personnes d'être acceptées et reconnues pour leurs esprit
spécial et unique.
- Therapeutique: Apprendre à guérir est une force puissante pour
la transformation et l'intégration. Un des grands points forts du leadership-
serviteur est son potentiel curatif sur soi-même et sur les autres.
- Honneteté: La conscience et particulièrement la conscience de soi-même générale,
renforce le leader-serviteur.
-Ideation: Les leaders-serviteurs cherchent à consolider leurs capaciter de "rêver des
grands rêves". La capacité de regarder un problème (ou une organisation) d'une perspective
conceptuelle signifie qu'on doit penser au-delà des réalités au jour le jour.

Source : conception des auteurs de l'article (Dounia BERE et Patrick ADON).

Ecoute : Le leader a un engagement profond à l'écoute attentive des autres. L'écoute également englobe : contacter sa propre voix intérieure et chercher à comprendre quelles parties du corps, de l'esprit ou de l'âme communiquent. L'écoute, associée à des périodes régulières de réflexion, est essentielle à la croissance du leaderserviteur.

Mentorat : Le leader-serviteur est profondément engagé dans la croissance de chaque individu dans son organisation. C'est en faisant grandir les autres que le Leader grandi. Il dépense toutes ses ressources (temps, énergies, finances etc.) pour aider les autres à grandir et à se développer.

Persuasion : Le leader-serviteur n'utilise pas l'autorité mais plutôt la persuasion pour diriger. Les suiveurs gagnés par persuasion sont plus fidèles que ceux qui n'obéissent que par contrainte.

Allocentrisme : Le leader-serviteur tâche de comprendre et de compatir avec les autres. Le besoin des personnes d'être acceptées et reconnues pour leur esprit spécial et unique.

Thérapeutique : Apprendre à guérir est une force puissante pour la transformation et l'intégration. Un des grands points forts du 
leadership-serviteur est son potentiel curatif sur soi-même et sur les autres. Au-delà du collaborateur, le leader serviteur doit s'intéresser à l'homme dans sa complexité.

Honnêteté : La conscience et particulièrement la conscience de soimême générale, renforce le leader-serviteur. La prise de conscience de ce que l'on est primordiale pour une collaboration avec autrui. : « La capacité de se manager soi-même, avoir la conscience de soi-même et s'autoréguler, est la base même pour manager les autres » a dit Daniel Goleman (1995).

Idéation : Les leaders-serviteurs cherchent à consolider leurs capacités de "rêver des grands rêves". La capacité de regarder un problème (ou une organisation) d'une perspective conceptuelle signifie qu'on doit penser au-delà des réalités quotidiennes. Il fait rêver ses suiveurs à travers des visions partagées et les fait sortir des sentiers battus.

Economat : Le leader préserve les intérêts de ses suiveurs. Il ne cherche pas l'attention mais en donne plutôt pour mettre les autres en confiance pour éclore leur dévouement.

Observés de manière parallèle, tous les termes que la désagrégation d'EMPATHIE a donné se trouvent avoir une équivalence sémantique parmi les 10 caractéristiques du Leadership serviteur présentés par L. Spears ${ }^{8}$. Dans le contexte de changement organisationnel, le leader se définirait donc comme un gestionnaire SMART qui fait preuve d'empathie pour les gens qu'il dirige. Les termes SMART et EMPATHIE de cette définition rendent à la fois les contenus des conclusions de GLOBE pour l'Afrique subsaharienne et les contenus des théories du leadership transformationnel et du leadership serviteur, tel que présentés par leurs concepteurs, donnant un paradigme de leadership plus complet, avec autant d'intérêt pour les objectifs de l'organisation que pour les personnes qui sont impactées par le changement.

En définitive, si une gestion de processus de changement doit avoir des résultats qui satisfassent l'organisation tout en lui conservant sa dignité à l'employé dans le contexte socioculturel de l'Afrique Subsaharienne tel que disséqué par l'étude de GLOBE ; une mixture des théories de Bernard Bass (1990, 1995) et de Robert Greenleaf (1977) est donc absolument nécessaire. L'alliage des styles de leadership et des présupposés culturels ne peut que correspondre aux exigences des acteurs en présence et aux exigences environnementales qui constituent un des facteurs clés de succès de gestion à maîtriser dans le contexte de l'Afrique subsaharienne francophone. C'est dire que la dynamique de l'organisation est plus fonction de la maitrise de ses

${ }^{8}$ Larry Spears, http://www.spearscenter.org/ 
variables socioculturelles et de son système de valeurs, que de ses indicateurs technico-économiques.

Si l'un des deux processus de réduction de personnel étudiés été jugé satisfaisant, c'est indéniablement grâce à la gestion efficace des aspects liés aux ressources humaines, et notamment à l'attention accordée aux sensibilités socioculturelles. L'adoption de bonnes stratégies, la participation des employés, la communication et le partage d'informations, le soutien apporté aux personnes touchées, la présentation de la vision et le leadership manifesté, l'administration digne et loyale des victimes, sont autant de facteurs importants de ce succès.

Il est néanmoins difficile de formuler une recommandation sur les facteurs de succès de la gestion du changement pouvant apparaître partout comme un bâton magique. La raison principale en est que chaque environnement d'exploitation est toujours différent. La géographie, les sociétés, les cultures, les réglementations et d'autres facteurs entrent en jeu. Ce qui est important, c'est que le responsable de la réduction des effectifs évalue le projet de manière critique et identifie les caractéristiques critiques pertinentes qui permettront d'obtenir d'excellents résultats.

\section{Conclusion}

Même si les problèmes inhérents au changement sont similaires d'une organisation à une autre, les spécificités du contexte et le type de leadership déployé rendent les résultats différents d'un processus à un autre. Ainsi, la façon de diriger le processus pourra avoir pour effet, soit d'atteindre de la manière la plus douce les objectifs escomptés, soit de générer des résistances pouvant conduire à l'échec du processus. Ce dualisme n'est que le résultat de la complexité des organisations et des hommes et femmes qui constituent les organisations. La recherche qui a été faite en utilisant à la fois des méthodes quantitatives et qualitatives de recherche, et en mettant à contribution les théories de leadership transformationnel, situationnel, serviteur a permis de déterminer un modèle « fétiche » de leadership pour le contexte considéré. Le modèle développé a pour atouts sa testabilité et sa validité empirique qui découlent des conclusions d'études empiriques qui la sous-tendent. Aussi, compte tenu de ces atouts, de son autonomie vis à vis de la littérature antérieure ou des observations empiriques antérieures, elle est potentiellement bien pour les recherches futures sur le leadership et le management en contexte africain subsaharien pour lequel les théories existantes semblent inappropriées ou peu fructueuses.

Le continent africain étant dominé par des thèses culturalistes et humanistes de gestion (Hofstede, 1980), sauf un type de leadership fortement humain, qui prend appui sur les indicateurs culturels (la nature des relations interpersonnelles, le rapport au temps, l'appartenance au milieu social 
traditionnel, etc.) peut assurer une réussite. Ces différents indicateurs confirment les postulats selon lesquels la culture africaine est basée sur la primauté du groupe sur l'individu, la primauté du social sur l'économique, et la nécessité de la convivialité sur l'efficacité.

L'apport de cette recherche sur cette question, qui a déjà été très largement traité dans de très nombreux articles et livres, par d'éminents chercheurs et spécialistes du leadership, porte plus sur la dimension du modèle adapté de leadership transformationnel qu'il propose, que sur l'importance des considérations culturelles dans la gestion ; cet aspect a déjà fait 1'objet d'une littérature abondante (Hernandez, 2007 ; Evralde Mutabazi, 2008 ; Patrick Bakengela Shamba, Yves Fréderic Livian, 2014). Les auteurs admettent avec Nollen (1996) qu'"il n'y a pas de management idéal. Les différences dans la culture nationale appellent à des différences dans les pratiques managériales". Cette recherche ne constitue qu'une modeste avancée dans la compréhension des réductions d'effectifs. L'étude de ces cas a permis de scruter les processus de réduction d'effectifs en organisations internationales à but non lucratif et d'évaluer les styles de leadership qui y avaient été manifestés. Il aurait été bien de faire le même examen sur les organisations à but lucratif mais les auteurs n'ont pas pu obtenir l'autorisation d'en étudier une. D'autres études empiriques sont nécessaires pour confirmer ce modèle et tendre vers sa théorisation. La réplication de la même étude sur des organisations à buts lucratifs avec un échantillon plus large dans le même contexte socioculturel serait la prochaine étape pour confirmer les caractéristiques identifiées.

\section{References:}

1. ANTONAKIS, J., \& HOUSE, R. J. (2002). An analysis of the fullrange leadership theory: The way forward. In B. J. Avolio \& F. J. Yammarino (Eds.) Transformational and Charismatic Leadership: The Road Ahead (pp. 3-34). Amsterdam: JAI Press.

2. ANTONAKIS, J., ANNA T. CIANCIOLO ET ROBERT J. STERNBERG, DIR., "The Nature of Leadership", Thousand Oaks, CA: Sage, Chap. 8

3. BAKENGELA SHAMBA, Patrick, LIVIAN, Yves Fréderic (2014). Le management africain introuvable : Pour une approche de l'hybridité segmentée. 4 conference ATLAS AFMI, May 2014, Marseille, France.

4. BAKENGELA SHAMBA, Patrick (2007). « existe-t-il un modèle spécifique du management en afrique ? Le management africain a l'épreuve des évidences empiriques ». 18eme congres de l'AGRH, Sep 2007, Fribourg, Suisse. Actes de congres de l'AGRH 2007, Université de Fribourg, Suisse.

5. BARBUTO, John E. and WHEELER, Daniel W. (2006). "Scale Development and Construct Clarification of Servant Leadership" . 
Faculty Publications: Agricultural Leadership, Education \& Communication Department. 51

6. BASS B. (1985). Leadership and performance beyond expectations. - New York: Free Press.

7. BASS, B. M. AVOLIO, B. J. (1994). Improving Organizational Effectiveness Through Transformational Leadership. Thousand Oaks, CA: Sage Publications

8. BELLET, D. (2016). Le leadership humainement intelligent de demain, Management Prospective Editions.

9. BELLET, D. (2013). L'évolution de l'industrie, Hachette Livre, 368 pages.

10. BURNS, J.M. (1978). Leadership. New York. Harper \& Row

11. CAMERON, K. S. (1994). Strategies for Successful Organizational Downsizing. Human Resource Management, 33, 189-211.

12. CROZIER, M. 1970). Early Evolutionary History of the Synapsida, edited by Christian F. Kammerer, Kenneth D. Angielczyk, Jörg Fröbisch

13. CROZIER, M. et FRIEDBERG, E. (1977). L'acteur et le système : les contraintes de l'action collective, Editions du Seuil, 436 p.

14. FIEDLER, F.E. (1967). A theory of leadership effectiveness. New York: McGraw-Hili.

15. HERZBERG, F.; MAUSNER, B.; SNYDERMAN, B. B. (1959). The Motivation to Work (2nd ed.). New York: John Wiley and Sons

16. GOLEMAN, D. (1995). Emotional Intelligence: Why It Can Matter More Than IQ, Bantam Books.

17. GREENLEAF, R. K. (1977). Servant leadership: A journey into the nature of legitimate power and greatness. New York: Paulist Press.

18. HAMILTON, C-A and NORRIE, J. (2003). The A to Z Guide to SoulInspiring Leadership, Epic Press, 270 pages.

19. HERNANDEZ, E-M. (1997). Le management des entreprises africaines. Essai de management du développement, Edition l'Harmattan, Paris.

20. HERNANDEZ E.M. (2000). L'actualité du modèle paternaliste, Revue Française de Gestion, mars, mai, pp. 85-105.

21. HOFSTEDE G. (1983). The cultural relativity of organizational practices and theories", Journal of International Business Studies, Vol. 14, No.2.

22. HOFSTEDE G. (1993). Cultural Constraints in Management Theories. - Academy of Management Executive, vol. 7, n 1, p. 81- 94.

23. HOFSTEDE, G (1980). "Culture and Organizations », International Studies of Management \& Organization, vol. 10, 1er janvier 1980. 
24. MASLOW,

A. (1943). A

Theory of

Human

Motivation », Psychological

Review, vol. $50, \mathrm{n}^{\mathrm{o}} 4$, juillet 1943, p. 370-396

25. MUTABAZI, E. (2005). Le Modèle Circulatoire de Management en Afrique. Revue Business Digest "Management de la diversité". Théories et pratiques innovantes en management et en Stratégie. Paris. $\mathrm{N}^{\circ} 157$, Novembre p. 24-26

26. PATTERSON, K. A. (2003). Servant Leadership: A Theoretical Model;

27. POLLARD, R.Q. (1996). Professional psychology and deaf people: The emergence of a discipline. American Psychologist 51(4)

28. SPEARS, Larry C. (2001). Focus on Leadership: Servant-Leadership for the 21st Century: Servant-leadership for the Twenty-first Century.

29. WILLETT, G. (2012). Paradigme, théorie, modèle, schéma : qu'est-ce donc ? », Communication et organisation

30. YAMMARINO, F. J., \& DANSEREAU, F. (2008). Multi-level nature of and multi-level approaches to leadership. Leadership Quarterly, 19, 135-141.

31. YAMMARINO, F. J., \& DANSEREAU, F. (2009). A new kind of OB (organizational behavior). Research in Multi-Level Issues, 8, 13-60.

32. YAMMARINO, F. J., \& DANSEREAU, F. (2011). Multi-level issues in evolutionary theory, organizational science, and leadership. Leadership Quarterly, 22, 1042-1057

33. YAMMARINO, F. J., DIONNE, S. D., CHUN, J. U., \& DANSEREAU, F. (2005). Leadership and levels of analysis: A stateof-the-science review. Leadership Quarterly, 16, 879-919.

34. YAMMARINO, F. J., SALAS, E., SERBAN, A., SHIRREFFS, K., \& SHUFFLER, M. L. (2012). Collectivistic leadership approaches: Putting the "we" in leadership science and practice. Industrial and Organizational Psychology: Perspectives on Science and Practice, 5, 382-402.

35. ZADI M. K, (1998). Culture africaine et management de l'entreprise moderne, Ed. Ceda, oct. 1998, Abidjan. 